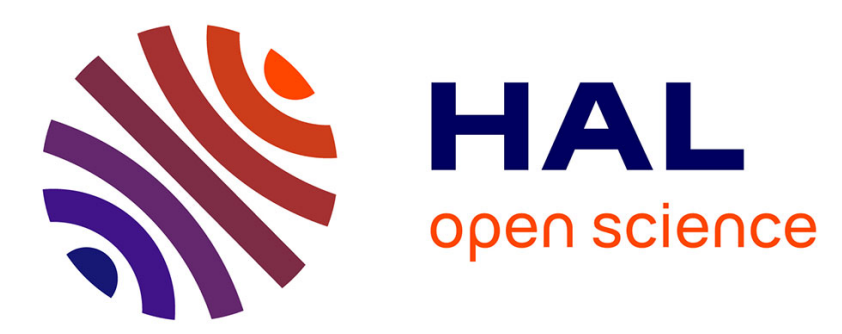

\title{
Quantifying foot deformation using finite helical angle
} Claude Pothrat, Benjamin Goislard de Monsabert, Laurent Vigouroux, Elke

Viehweger, Eric Berton, Guillaume Rao

\section{To cite this version:}

Claude Pothrat, Benjamin Goislard de Monsabert, Laurent Vigouroux, Elke Viehweger, Eric Berton, et al.. Quantifying foot deformation using finite helical angle. Journal of Biomechanics, 2015, 48 (13), pp.3716-3719. 10.1016/j.jbiomech.2015.07.044 . hal-01414105

\section{HAL Id: hal-01414105 \\ https://hal.science/hal-01414105}

Submitted on 17 Nov 2017

HAL is a multi-disciplinary open access archive for the deposit and dissemination of scientific research documents, whether they are published or not. The documents may come from teaching and research institutions in France or abroad, or from public or private research centers.
L'archive ouverte pluridisciplinaire HAL, est destinée au dépôt et à la diffusion de documents scientifiques de niveau recherche, publiés ou non, émanant des établissements d'enseignement et de recherche français ou étrangers, des laboratoires publics ou privés. 
Short communication

\title{
Quantifying foot deformation using finite helical angle
}

\author{
Claude Pothrat ${ }^{\mathrm{a}, *}$, Benjamin Goislard de Monsabert ${ }^{\mathrm{a}}$, Laurent Vigouroux ${ }^{\mathrm{a}}$, \\ Elke Viehweger $^{\mathrm{a}, \mathrm{b}}$, Eric Berton ${ }^{\mathrm{a}}$, Guillaume Rao ${ }^{\mathrm{a}}$ \\ a Aix-Marseille University, CNRS, ISM UMR 7287, 163 avenue de Luminy, 13288 Marseille cedex 09, France \\ ${ }^{\mathrm{b}}$ APHM, Hopital La Timone, Service d'orthopédie pédiatrique, 13005 Marseille, France
}

\section{A R T I C L E I N F O}

\section{Article history:}

Accepted 30 July 2015

\section{Keywords:}

Foot kinematics

Repeatability

Sport movements

\begin{abstract}
A B S T R A C T
Foot intrinsic motion originates from the combination of numerous joint motions giving this segment a high adaptive ability. Existing foot kinematic models are mostly focused on analyzing small scale foot bone to bone motions which require both complex experimental methodology and complex interpretative work to assess the global foot functionality. This study proposes a method to assess the total foot deformation by calculating a helical angle from the relative motions of the rearfoot and the forefoot. This method required a limited number of retro-reflective markers placed on the foot and was tested for five different movements (walking, forefoot impact running, heel impact running, $90^{\circ}$ cutting, and $180^{\circ}$ U-turn) and 12 participants. Overtime intraclass correlation coefficients were calculated to quantify the helical angle pattern repeatability for each movement. Our results indicated that the method was suitable to identify the different motions as different amplitudes of helical angle were observed according to the flexibility required in each movement. Moreover, the results showed that the repeatability could be used to identify the mastering of each motion as this repeatability was high for well mastered movements. Together with existing methods, this new protocol could be applied to fully assess foot function in sport or clinical contexts.
\end{abstract}

(c) 2015 Elsevier Ltd. All rights reserved.

\section{Introduction}

The foot is a central element for locomotion and is subject to repetitive and intensive loadings during daily life. Foot musculoskeletal disorders therefore frequently occur and could lead to painful affections of the knee and hip joints, and back segment (Bird and Payne, 1999). Understanding foot movements is crucial to improve rehabilitation programs, surgical decision-making, or athletic performance. The foot contains numerous small bones with relative motions around multiple axes, ranging between $5^{\circ}$ and $15^{\circ}$ during slow running (Arndt et al., 2007), which combination results in the total foot deformation. The foot's specific architecture renders in a good ability to adapt ground unevenness and resist cyclic or non-cyclic loadings. Because of the complexity of the foot joint structure, the small ranges of motion and the small size of foot bones, the analysis of intrinsic foot kinematics has always been a challenge.

Existing models of foot kinematics tend be more and more detailed, sometimes using medical imaging measurements (Beimers et al., 2008; Gutekunst et al., 2013; Ledoux et al., 2006; Stindel et al., 1999a, 1999b; Woodburn et al., 2002) or including up to nine segments tracked with 19 external markers (Arndt et al.,

\footnotetext{
* Corresponding author. Tel.: +33 4917596 56; fax: +33 491172252.

E-mail address: claude.pothrat@univ-amu.fr (C. Pothrat).
}

2007; Baker and Robb, 2006; Deschamps et al., 2011; Leardini et al., 1999; Nester et al., 2010; Simon et al., 2006; Stebbins et al., 2006). Most of these models calculate joint kinematics using Cardan rotation sequences as recommended by ISB (Wu and Cavanagh, 1995). This method is however subject to controversy (Chèze, 2000; Piazza and Cavanagh, 2000) as its outputs are known to be prone to error propagation (Wu et al., 2002) and subject to cross talk effects (Phadke et al., 2011; Ramsey and Wretenberg, 1999). Moreover, given the high number of embedded degrees of freedom in the foot, the three independent orthogonal angles from the Cardan sequence are unlikely to anatomically represent the complex multidirectional foot intrinsic motion.

As an alternative approach to the Cardan method, the finite helical axis (FHA) allows to represent movements between two segments while remaining independent from the orientation of local embedded coordinate systems (Marin et al., 2003; Sheehan, 2007; Woltring, 1994). Numerous studies on knee (Blankevoort et al., 1990; Marin et al., 2003; Sheehan, 2007; van den Bogert et al., 2008), spine (Kettler et al., 2004), neck (Woltring et al., 1994), ankle (Tuijthof et al., 2009) joints and foot bone-to-bone description showed the interest of using the FHA to clarify joint rotation axes or movement kinematics (Arndt et al., 2007). Graf and Stefanyshyn (2012a, 2012b) and Graf et al. (2012) also used the helical axis to analyze the main orientation around which the foot internal movement occur. However, this study did not consider the 
helical angle value in itself, while this angle may be interesting to quantify the total intrinsic foot motion during several tasks. Although these previous methods brought a significant contribution to the understanding of foot motion, the application of the helical angle to assess total foot movement can be widened.

The present study aimed to propose a complementary method to the existing ones (Graf et al., 2012; Stebbins et al., 2006), to assess foot global deformation during daily and sport movements. The helical angle will be calculated to express the movement between forefoot and rearfoot segments during five different movements. The method will be statistically analyzed in terms of repeatability. We hypothesized that the helical angle is representative of foot total intrinsic movement and provides a characterization of each movement.

\section{Methods}

\subsection{Subjects and tasks}

Twelve subjects, 6 men and 6 women (mean \pm sd: $26.6 \pm 4.4$ years; $66.5 \pm 8.2 \mathrm{~kg} ; 174.7 \pm 6.1 \mathrm{~cm}$ ) with no lower limb injury volunteered for this study. All participants gave informed consent and the local ethical committee has approved the protocol. Following a training period, each subject performed five repetitions of five different movements: walking, running with forefoot impact, running with heel impact, $90^{\circ}$ lateral cutting, and $180^{\circ}$ U-turn in a randomized order (see Supplementary material for more details). All movements were performed along a $12 \mathrm{~m}$ pathway, equipped with an embedded force plate (Kistler, model $9281,1000 \mathrm{~Hz}$ ) located $7 \mathrm{~m}$ after the start of the pathway.

\subsection{Data acquisition and analysis}

Six retro-reflective markers were placed on the forefoot and rearfoot parts of the right foot segment following the protocol of Graf et al. (2012) ( Fig. 1a and b, Supplementary material).

Kinematic data were recorded by an 8-camera optoelectronic system (Vicon, Oxford UK, $125 \mathrm{~Hz}$ ). Ground reaction forces and kinematics data were synchronized and acquired using the Vicon system. Data from the force plate were used to precisely determine the stance phase. Kinematic data were filtered using a zero time-lag 4th order low-pass Butterworth (net cut-off frequency: $6 \mathrm{~Hz}$ ), then time normalized on 100 points over the stance phase.

Right-handed local coordinates systems (CS) were constructed and the relative motion of the forefoot relative to the rearfoot CS was quantified by calculating the helical angle for each time point of the movement (Supplementary material). Results have been offset by the value of the helical angle at the instant preceding the impact. The total waveform of the helical angle, quantifying the global intrinsic foot motion, was studied as well as two finite variables: its maximum and amplitude.

\subsection{Statistical analysis}

Repeated measures ANOVAs were performed on two finite variables (helical angle maximum and amplitude) separately for each task in order to evaluate their capacity to discriminate in between the five conditions and therefore to be representative of foot intrinsic global deformation. In addition, classical ICC was calculated on these variables to estimate their repeatability (Mahaffey et al., 2013) and may validate the choice of these finite variables to represent the total foot deformation. Overtime intra-class correlation coefficients (ICCX) were calculated to assess the helical angle repeatability over the entire waveform based on Duhamel's method (Duhamel et al., 2004; Preatoni et al., 2013) (see Supplementary material). ICCX is expressed between 0 and 1 . Shrout's interpretation has been retained for the evaluation of our measures (Shrout, 1998) as follows: between 0 and 0.1: no repeatability; between 0.11 and 0.4 : slight; between 0.41 and 0.6 : fair; between 0.61 and 0.8: moderate; between 0.81 and 1 : substantial. Repeated measures ANOVAs were performed on the ICCX, with the 12 participants as observations and the five movements as conditions. Results were considered significant when $p<0.05$ and Newman-Keuls post-hoc test were used when necessary.

\section{Results}

Averaged maximum values of the helical angle ranged from $4.43 \pm 4.5^{\circ}$ for the $180^{\circ} \mathrm{U}$-turn to $20.78 \pm 6.65^{\circ}$ for walking (Figs. 2

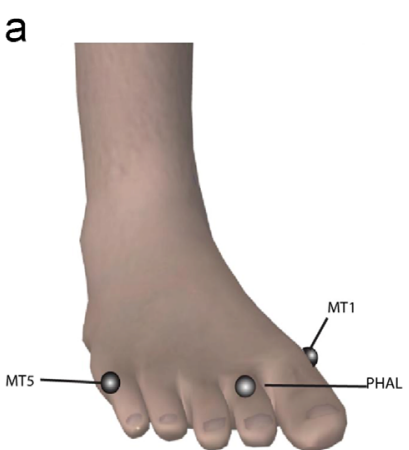

Forefoot markers positioning

C

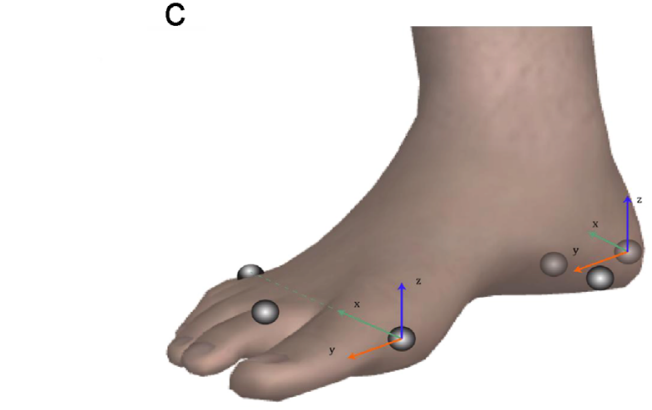

coordinate systems b

Rearfoot markers positioning

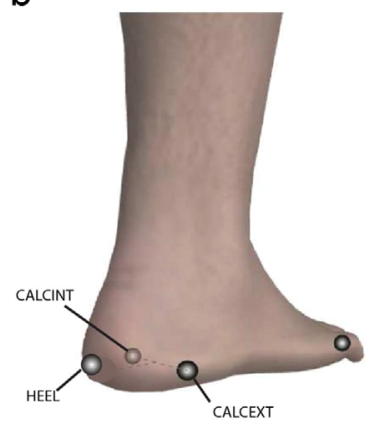

Fig. 1. (a, b) Markers positioning used for the finite helical angle (FHA) calculations. (a) Forefoot markers. One marker is placed on the first metatarsal head (MT1), one is placed on the fifth metatarsal head (MT5), and the third is placed on the first phalange of the first toe (PHAL). (b) Rearfoot markers. Two markers are placed respectively on the lateral (CALCEXT) and medial (CALCINT) aspects of the calcaneum. The third one is placed on the posterior aspect pf the calcaneum (HEEL). (c) Forefoot and rearfoot coordinate systems. The FHA will be calculated as the resolution of the rotations between these two coordinate systems.

and 3), maximum helical angle was significantly different between all the conditions $\left(F_{4,50}=21.7 p<0.05\right)$ except for $90^{\circ}$ cutting $\left(12.54 \pm 7.8^{\circ}\right)$ similar to heel impact running $\left(13.01 \pm 6.72^{\circ}\right)$ (Table 1$) .180^{\circ} \mathrm{U}$-turn was characterized by the smallest amplitude $\left(9.9 \pm 4.13^{\circ}\right)$ and walking revealed the biggest amplitude with $26.5 \pm 5.23^{\circ}$ (Figs. 2 and 3; Table 1). All movement amplitudes were significantly different from each other $(p<0.05)$. The ICCs on finite variables showed values higher than 0.7 for all movements for both the maximum and the amplitude variables, except for the $\mathrm{U}$ turn movement, for which both of the variables had an ICC under 0.6 (Table 1 ).

The intraclass correlation coefficients showed significant differences in between the movements. The three daily-performed movements: walking and two types of running showed ICCX respectively equal to $0.95 \pm 0.07,0.97 \pm 0.02$, and $0.95 \pm 0.05$ meaning a substantial repeatability, $90^{\circ}$ cutting obtained an ICCX equal to $0.84 \pm 0.15$ and $180^{\circ}$ U-turn had ICCx equal to $0.37 \pm 0.36$. The ICCx of the three daily-performed movements and the $90^{\circ}$ cutting were significantly different from the ICC of the $180^{\circ} \mathrm{U}$-turn movements $\left(F_{4,50}=19.85, p<0.05\right)$ without significantly differing from each other (Fig. 3 ).

\section{Discussion}

This study aimed at proposing a complementary method to existing ones (Graf et al., 2012; Stebbins et al., 2006) to assess overall foot deformations during dynamic activities. Based on existing methods that looked at the main axis of motion within the foot, the present approach quantifies the helical angle between the 
forefoot and the rearfoot segments in order to assess the global amount of deformation instead of considering multiple articular joint angles. This approach has the advantage of being easily implemented to a high number of locomotor tasks and to different populations because of the small number of required markers $(n=6)$. Particularly, the marker placement proposed by Graf et al. and used in the present study is compatible with sports or clinical contexts. Furthermore, since the FHA is not sensible to the orientation of embedded local frames, the proposed method is not very sensitive to marker placement and could be used with other motion capture tools such as inertial sensors.

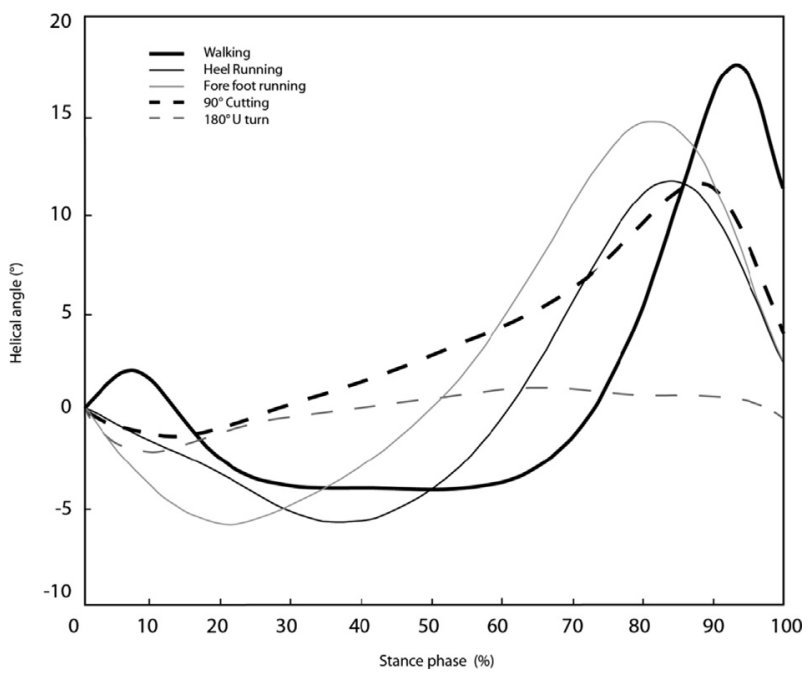

Fig. 2. Averaged helical angle profiles for the five experimental conditions, time normalized over the stance phase. Results have been offseted by the value of the helical angle at the instant preceding the impact.

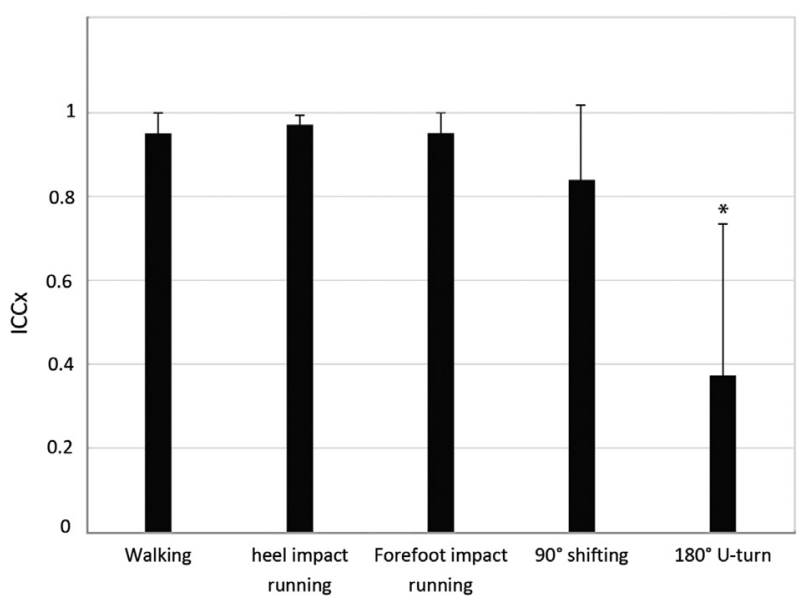

Fig. 3. Averaged ( \pm s.d.) overall cycle ICCx values for each movement. * represents significant differences from all other conditions $(p<0.05)$.
In addition to previous existing methodologies, the main contribution of our method, while not providing a complete description of the motion, is to consider the total amount of intrinsic foot deformation in order to fully characterize foot function and is complementary to existing methodologies. Based on the results observed for five different motions, the method appeared suitable for discriminating the different motions as the computed variables were significantly different when comparing a specific activity with the other ones (Table 1, Fig. 3). Moreover, the classical ICC performed on the finite variables (e.g. maximum and amplitude values of the helical angle) showed repeatable results for all the movements (Table 1). The present method can therefore give reliable information on foot total deformation during either cyclic or non-cyclic movements. Maximum and amplitude of the helical angle were representative of foot intrinsic deformation and sufficiently sensitive to statistically differentiate movements between them. As expected, cyclic locomotor movements that require more flexibility from the foot reached the highest values of the helical angle. Opposite to that, non-cyclical movements as $90^{\circ}$ cutting and the U-turn got less amplitude, related to a more stiffened foot that could correspond to a specific postural control of the foot to avoid injury or increase performance (Kelly et al., 2014). Interestingly, using overtime ICCx to assess the repeatability of the helical angle over the stance phase of each movement can be used to identify a signature of movement expertize. The repeatability of the helical angle for well-known locomotor tasks (walking and running) and $90^{\circ}$ cutting was all higher than for the U-turn, reflecting poor mastering of the latter from the participants. Therefore, the use of overtime ICCX on the helical angle seems to be a new objective tool for understanding the foot deformation under different loads, or the effects of training on this deformation over time.

More than the usual interpretation of standard deviation values (Stebbins et al., 2006), the use of ICC and ICCX methods can become a standard for the evaluation of the multisegments foot model repeatability and further be compared to the proposed method that already showed fairly good results in this topic.

When considering the present results and method, some limitations should be considered. First, kinematic analyses are subject to inherent limitations such as the use of external markers which are affected by skin motion artifact. Second, this method breaks away from traditional analysis and does not describe the motion in terms of flexion/extension, abduction adduction and external/ internal rotation movements. As these three rotations are known to be relevant in clinical context, an adaptation of our method could be further implemented by projecting the helical axis in the rearfoot coordinate system and analyzing the components of the helical angle.

To conclude, the proposed method proved capable of characterizing overall foot deformation through the helical angle, thereby being complementary to existing methods. The use of helical angle appeared a suitable method to quantify foot deformation and therefore to improve our knowledge of foot intrinsic deformation during numerous tasks. It would be interesting to investigate on the information that this angle could bring on foot function disorders associated with pathological deformation or to

Table 1

Maximum and amplitude values of the finite helical angle (mean \pm standard deviation) for the five experimental movements. ICC is presented for each variable.

\begin{tabular}{|c|c|c|c|c|c|c|c|c|c|c|}
\hline \multirow[t]{2}{*}{ Variable } & \multirow{2}{*}{$\begin{array}{l}\text { Walking } \\
\text { Value (deg) }\end{array}$} & \multirow[b]{2}{*}{ ICC } & \multicolumn{2}{|l|}{ Heel impact run } & \multicolumn{2}{|c|}{ Fore foot impact run } & \multicolumn{2}{|l|}{$90^{\circ}$ shifting } & \multicolumn{2}{|l|}{$180^{\circ}$ U-turn } \\
\hline & & & Value (deg) & ICC & Value (deg) & ICC & Value (deg) & ICC & Value (deg) & ICC \\
\hline Maximum & $20.78 \pm 6.65^{a}$ & 0.76 & $13.01 \pm 6.72$ & 0.88 & $16.4 \pm 8.37^{\mathrm{a}}$ & 0.82 & $12.54 \pm 7.80$ & 0.79 & $4.42 \pm 4.58^{\mathrm{a}}$ & 0.52 \\
\hline Amplitude & $26.49 \pm 5.23^{a}$ & 0.70 & $20.17 \pm 4.13^{\mathrm{a}}$ & 0.72 & $23.64 \pm 5.48^{\mathrm{a}}$ & 0.83 & $16.45 \pm 4.97^{a}$ & 0.82 & $9.86 \pm 4.13^{\mathrm{a}}$ & 0.31 \\
\hline
\end{tabular}

a The variable for the considered condition is significantly different from all other conditions. 
what extent it could characterize a difference between different foot types.

\section{Conflict of interest statement}

All authors disclose that there was no conflict of interest regarding this study.

\section{Appendix A. Supplementary material}

Supplementary data associated with this article can be found in the online version at http://dx.doi.org/10.1016/j.jbiomech.2015.07.044.

\section{References}

Arndt, A., Wolf, P., Liu, A., Nester, C., Stacoff, A., Jones, R., Lundgren, P., Lundberg, A. 2007. Intrinsic foot kinematics measured in vivo during the stance phase of slow running. J. Biomech. 40, 2672-2678.

Baker, R., Robb, J., 2006. Foot models for clinical gait analysis. Gait Posture 23, 399-400.

Beimers, L., Tuijthof, G.J.M., Blankevoort, L., Jonges, R., Maas, M., Van Dijk, C.N., 2008. In-vivo range of motion of the subtalar joint using computed tomography. J. Biomech. 41, 1390-1397.

Bird, A.R., Payne, C.B., 1999. Foot function and low back pain. Foot 9, 175-180.

Blankevoort, L., Huiskes, R., De Lange, A., 1990. Helical axes of passive knee joint motions. J. Biomech. 23, 1219-1229.

Chèze, L., 2000. Comparison of different calculations of three-dimensional joint kinematics from video-based system data. J. Biomech. 33, 1695-1699.

Deschamps, K., Staes, F., Roosen, P., Nobels, F., Desloovere, K., Bruyninckx, H., Matricali, G.A., 2011. Body of evidence supporting the clinical use of 3D multisegment foot models: a systematic review. Gait Posture 33, 338-349.

Duhamel, A., Bourriez, J.L., Devos, P., Krystkowiak, P., Destée, A., Derambure, P., Defebvre, L., 2004. Statistical tools for clinical gait analysis. Gait Posture 20, 204-212.

Graf, E.S., Stefanyshyn, D.J., 2012a. The shifting of the torsion axis of the foot during the stance phase of lateral cutting movements. J. Biomech. 45, 2680-2683.

Graf, E.S., Stefanyshyn, D.J., 2012b. Average torsion axis location of atletic movements: subject specific or movement specific? J. Appl. Biomech. 29 (3), 365-368.

Graf, E.S., Wright, I.C., Stefanyshyn, D.J., 2012. Effect of relative marker movement on the calculation of the foot torsion axis using a combined Cardan angle and helical axis approach. Comput. Math. Methods Med. 2012, 368050.

Gutekunst, D.J., Liu, L., Ju, T., Prior, F.W., Sinacore, D.R., 2013. Reliability of clinically relevant 3D foot bone angles from quantitative computed tomography. J. Foot Ankle Res. 6, 38

Kelly, L.A., Lichtwark, G., Cresswell, A.G., 2014. Active regulation of longitudinal arch compression and recoil during walking and running. J. R. Soc. Interface 12, 20141076.

Kettler, A., Marin, F., SattleMayer, G., Mohr, M., Mannel, H., Durselen, L.J.W., 2004. Finite helical axes of motion are useful tool to describe the three dimensional in vitro kinematics of the intact, injured and stabilised spine. Eur. Spine J. 13, $553-559$.
Leardini, A., Benedetti, M.G., Catani, F., Simoncini, L., Giannini, S., 1999. An anatomically based protocol for the description of foot segment kinematics during gait. Clin. Biomech. 14, 528-536.

Ledoux, W.R., Rohr, E.S., Ching, R.P., Sangeorzan, B.J., 2006. Effect of foot shape on the three-dimensional position of foot bones. J. Orthop. Res., 21-23.

Mahaffey, R., Morrison, S.C., Drechsler, W.I., Cramp, M.C., 2013. Evaluation of multisegmental kinematic modelling in the paediatric foot using three concurrent foot models. J. Foot Ankle Res. 6, 43.

Marin, F., Mannel, H., Claes, L., Dürselen, L., 2003. Correction of axis misalignment in the analysis of knee rotations. Hum. Mov. Sci. 22, 285-296.

Nester, C.J., Liu, A.M., Ward, E., Howard, D., Cocheba, J., Derrick, T., 2010. Error in the description of foot kinematics due to violation of rigid body assumptions. J. Biomech. 43, 666-672.

Phadke, V., Braman, J.P., LaPrade, R.F., Ludewig, P.M., 2011. Comparison of glenohumeral motion using different rotation sequences. J. Biomech. 44, 700-705.

Piazza, S.J., Cavanagh, P.R., 2000. Measurement of the screw-home motion of the knee is sensitive to errors in axis alignment. J. Biomech. 33, 1029-1034.

Preatoni, E., Hamill, J., Harrison, A.J., Hayes, K., Van Emerik, R.E., Wilson, C., Rodano, R., 2013. Movement variability and skills monitoring in sports. Sports Biomech. $12,69-92$.

Ramsey, D.K., Wretenberg, P.F., 1999. Biomechanics of the knee: methodological considerations in the in vivo kinematic analysis of the tibiofemoral and patellofemoral joint. Clin. Biomech. 14, 595-611.

Sheehan, F.T., 2007. The finite helical axis of the knee joint (a non-invasive in vivo study using fast-PC MRI). J. Biomech. 40, 1038-1047.

Shrout, P., 1998. Measurement reliability and agreement in psychiatry. Stat. Methods Med. Res. 7, 301-317.

Simon, J., Doederlein, L., McIntosh, A.S., Metaxiotis, D., Bock, H.G., Wolf, S.I., 2006. The Heidelberg foot measurement method: development, description and assessment. Gait Posture 23, 411-424.

Stebbins, J., Harrington, M., Thompson, N., Zavatsky, A., Theologis, T., 2006. Repeatability of a model for measuring multi-segment foot kinematics in children. Gait Posture 23, 401-410.

Stindel, E., Udupa, J., Hirsch, B., Odhner, D., 1999a. A characterization of the geometric architecture of the peritalar joint complex via MRI: an aid to the classification of foot type. IEEE Trans. Med. Imaging 18, 753-763.

Stindel, E., Udupa, J.K., Hirsch, B.E., Odhner, D., Couture, C., 1999b. 3D MR image analysis of the morphology of the rear foot: application to classification of bones. Comput. Med. Imaging Graph. 23, 75-83.

Tuijthof, G.J.M., Zengerink, M., Beimers, L., Jonges, R., Maas, M., van Dijk, C.N., Blankevoort, L., 2009. Determination of consistent patterns of range of motion in the ankle joint with a computed tomography stress-test. Clin. Biomech. 24, 517-523.

Van den Bogert, A.J., Reinschmidt, C., Lundberg, A., 2008. Helical axes of skeletal knee joint motion during running. J. Biomech. 41, 1632-1638.

Woltring, H.J., 1994. 3D attitude representation of human joint a standardization proposal. J. Biomech. 27, 1399-1414.

Woltring, H.J., Long, K., Osterbauer, P.J., Fuhr, A.W., 1994. Instantaneous helical axis estimation from 3-D video data in neck kinematics for whiplash diagnostics. J. Biomech. 27 (12), 1415-1432.

Woodburn, J., Udupa, J.K., Hirsch, B.E., Wakefield, R.J., Helliwell, P.S., Reay, N., O'Connor, P., Budgen, A., Emery, P., 2002. The geometric architecture of the subtalar and midtarsal joints in rheumatoid arthritis based on magnetic resonance imaging. Arthritis Rheum. 46, 3168-3177.

Wu, G., Cavanagh, P.R., 1995. ISB recommendation for standardization in reporting of kinematic data. J. Biomech. 28, 1257-1261.

Wu, G., Siegler, S., Allard, P., Kirtley, C., Leardini, A., Rosenbaum, D., Whittle, M., D'Lima, D.D., Cristofolini, L., Witte, H., Schmid, O., Stokes, I., 2002. ISB recommendation on definitions of joint coordinate system of various joints for the reporting of human joint motion-part I: ankle, hip and spine. J. Biomech. 35, 543-548. 